\title{
U-shaped associations between changes in blood pressure during dialysis and patient survival
}

B oth intradialytic hypertension and intradialytic hypotension are common complications of haemodialysis that have been reported to be associated with adverse outcomes. However, the extent to which changes in blood pressure levels during haemodialysis sessions affect patient outcomes is not yet clear. In a retrospective cohort study recently published in Kidney International, Jongha Park and colleagues report U-shaped associations between mean changes in intradialytic systolic and diastolic blood pressure levels and patient survival.

To evaluate the effect of changes in blood pressure levels during haemodialysis sessions on patient survival, the researchers analysed changes in intradialytic systolic and diastolic blood pressure (defined as the blood pressure level after the dialysis session minus the blood pressure level before the dialysis session) in a cohort of 113,255 US patients who were enrolled in the study between 1 July 2001 and 30 June 2006. They then assessed whether mean changes in intradialytic blood pressure (that is, the mean of the intradialytic blood pressure change values recorded for serial haemodialysis sessions during the follow-up period) were associated with patient mortality. "In order to more accurately and flexibly model the association between mean intradialytic blood pressure change and mortality, we employed Cox regression models using restricted cubic splines," say Park and coauthor Kamyar Kalantar-Zadeh. "These models do not assume a linear relationship between a predictor and an outcome, so enable detection of U-shaped and J-shaped associations-for example, an increase in mortality that is associated with both increases and decreases in blood pressure."

The researchers report that 53,461 study participants died during a median follow-up of 2.2 years; 21,548 deaths were

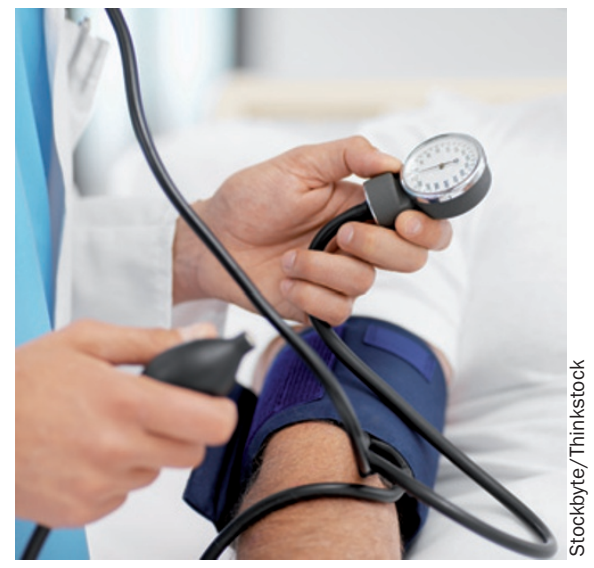

the result of cardiovascular causes. They found that the mean intradialytic changes in systolic and diastolic blood pressure levels for the overall cohort during the follow-up period were $-10 \mathrm{mmHg}$ and $-5 \mathrm{mmHg}$, respectively. Compared with patients whose mean intradialytic change in systolic blood pressure was $-10 \mathrm{mmHg}$ to $<10 \mathrm{mmHg}$, those whose mean systolic blood pressure increased by more than $10 \mathrm{mmHg}$ during dialysis were older and had a higher prevalence of diabetes mellitus, ischaemic heart disease and congestive heart failure, whereas those whose mean systolic blood pressure decreased by $\geq 30 \mathrm{mmHg}$ were younger and had a higher prevalence of diabetes but a lower prevalence of ischaemic heart disease and congestive heart failure.

In unadjusted models and in models adjusted for sociodemographic factors, comorbidities and laboratory covariates, the researchers found U-shaped associations between mean intradialytic changes in systolic and diastolic blood pressure levels and all-cause mortality that were independent of blood pressure levels before the treatment sessions. In their fully adjusted model, mean intradialytic decreases in systolic blood pressure of $0-30 \mathrm{mmHg}$ and mean intradialytic changes in diastolic blood pressure of $-15 \mathrm{mmHg}$ to $5 \mathrm{mmHg}$ were associated with increased survival, but decreases in systolic blood pressure $>30 \mathrm{mmHg}$ and increases in systolic blood pressure during haemodialysis sessions were associated with increased all-cause mortality. Further analysis showed that the positive association between increases in mean systolic blood pressure during haemodialysis and all-cause mortality was restricted to patients whose systolic blood pressure prior to the treatment sessions was $\geq 120 \mathrm{mmHg}$. Mean intradialytic decreases in systolic blood pressure of $14 \mathrm{mmHg}$ (adjusted HR 0.92; 95\% CI 0.91-0.93) and in diastolic blood pressure of $6 \mathrm{mmHg}$ (adjusted HR 0.93; 95\% CI 0.91-0.94) were associated with the greatest patient survival. The researchers also observed U-shaped associations between mean changes in intradialytic systolic and diastolic blood pressure levels and cardiovascular mortality in adjusted and unadjusted models.

"Modest declines in blood pressure during haemodialysis sessions are associated with greater survival, whereas large declines in blood pressure (that is, dramatic intradialytic hypotension) or any rise in blood pressure (that is, intradialytic hypertension) are associated with increased mortality," conclude Park and Kalantar-Zadeh. They suggest that change in blood pressure levels during haemodialysis sessions could be a therapeutic target for improving patient outcomes but state that clinical trials are required to evaluate whether modest decreases in blood pressure during these sessions could improve patient survival and to find effective strategies that promote haemodynamic stability during haemodialysis.

Ellen F. Carney 